\title{
An Analytical Estimate of the Hubble Constant
}

\author{
Naser Mostaghel \\ Dept. of Civil Engineering, University of Toledo, Ohio, USA
}

\section{Email address:}

naser.mostaghel@utoledo.edu

\section{To cite this article:}

Naser Mostaghel. An Analytical Estimate of the Hubble Constant. American Journal of Astronomy and Astrophysics.

Vol. 3, No. 3, 2015, pp. 44-49. doi: 10.11648/j.ajaa.20150303.13

\begin{abstract}
Currently the present-time value of the Hubble constant is estimated through finding the optimum fit to the observationally measured data. Here, assuming a flat universe with zero cosmological constant, based on the conservation of total mass-energy and a correction for the effect of time dilation, the total present-time value of the energy density parameter is found to be equal to 0.703091. Based on the Friedmann-Robertson-Walker (FRW) equation, the first law of thermodynamics, and Einstein's Equivalence Principle, we present an analytical approach which yields a value for the Hubble constant equal to $H_{0}=69.05398 \mathrm{~km} \mathrm{~s}^{-1} \mathrm{Mpc}^{-1}$. Using this value, Hubble diagrams are constructed. These diagrams are remarkably consistent with the available observational data.
\end{abstract}

Keywords: Hubble Constant, Density Parameter, Distances and Redshift, Expanding Universe

\section{Introduction}

The present-time value of the Hubble constant provides a simple way to estimate the age and the size of the universe. As such the task of its accurate determination has been ardently undertaken by many teams of cosmologists since its introduction by Edwin Hubble [1] in 1929. Its determination involves precise measurements of redshift and of distances of far away galaxies. A galaxy's redshift is acquired through spectroscopic observations. But the accurate measurement of its distance is much more difficult. Freedman and Madore (in 2010) give details of six high-precision methods for the determination of distance, including the details of the known systematic errors in the measurement [2]. Tammann (in 2005) gives a detailed history of the determination of the Hubble constant and provides a table which includes 96 values for the Hubble constant varying from 50 to $109 \mathrm{~km} \mathrm{~s}^{-1} \mathrm{Mpc}^{-1}$ as evaluated by various teams of investigators from 1974 to 2005 [3]. Falcon and Aguirre (in 2014) have analytically evaluated a maximum value for the Hubble constant equal to $86.31 \mathrm{~km} \mathrm{~s}^{-1} \mathrm{Mpc}^{-1}$ [4]. The most recent estimated values based on observational data are: as reported by the SevenYear Wilkinson Microwave Anisotropy Probe, (in 2011), 71.0 $\pm 2.5 \mathrm{~km} \mathrm{~s}^{-1} \mathrm{Mpc}^{-1}$ [5]; as reported by the Planck Mission (in 2013), $67.8 \pm 0.77 \mathrm{~km} \mathrm{~s}^{-1} \mathrm{Mpc}^{-1}$ [6]; as reported (in 2013) by the Nine-Year Wilkinson Microwave Anisotropy Probe (WMAP), $69.32 \pm 0.80 \mathrm{~km} \mathrm{~s}^{-1} \mathrm{Mpc}^{-1}$ [7]; and as reported (in 2013) by the Megamaser Cosmology Project IV, $68.9 \pm 7.1 \mathrm{~km} \mathrm{~s}^{-1} \mathrm{Mpc}^{-1}[8]$.
In all of the above investigations, except the one by Falcon and Aguirre [4], the present-time value of the Hubble constant is determined empirically through an optimum fit to the observational data. Here, we assume a flat universe with zero cosmological constant. Based on the universe's total mass-energy conservation, we show that at the initiation of expansion of space the total energy divides into two parts. Considering both parts of the energy to be responsible for the expansion of space, and including the effect of time dilation on the rate of expansion, we find the total energy density parameter at the present time to be equal to 0.703091 . Using this value for the total energy density parameter and based on the Friedmann-Robertson-Walker (FRW) [9] equation, the first law of thermodynamics, and Einstein's Equivalence Principle, we present an analytical approach which yields the value for the Hubble constant equal to $H_{0}=69.05398 \mathrm{~km} \mathrm{~s}^{-1} \mathrm{Mpc}^{-1}$. This value is in between the ones estimated by the Nine-Year Wilkinson Microwave Anisotropy Probe (WMAP) and the Megamaser Cosmology Project IV team.

In the next section we develop the relations for the mass and energy fractions and we evaluate the total energy density parameter. The evaluations of the components of density parameters are presented in section 3. The details of the analytical derivation of the present-time value of the Hubble constant and the evaluation of its numerical value are presented in section 4 . The efficacy of the proposed approach and comparison with observational data are discussed in section 5 . 


\section{Mass and Energy Fractions}

In our approach to develop an analytical estimate of the Hubble constant, we first need to define fractions of mass and energy at the initiation of the big bang. To this end, we assume the universe to be flat, isotropic, and with a homogeneous distribution of mass and energy. Let $M_{t}$ represent the total mass of the universe including the total mass of all forms of ordinary and non-ordinary masses as well as the total equivalent mass of all energies at the start of the big bang. Thus

$$
M_{t}=M_{m}+M_{r}\left(t_{i n i}\right)
$$

where $M_{m}$ represents the total of ordinary and non-ordinary masses, excluding the equivalent mass of all energies, and $M_{r}\left(t_{\text {ini }}\right)$ represents the equivalent mass of all energies at the start of the big bang. If we represent $M_{r}\left(t_{i n i}\right)$ and $M_{m}$ in the following form

$$
\begin{gathered}
M_{r}\left(t_{\text {ini }}\right)=\gamma M_{t} \\
M_{m}=(1-\gamma) M_{t}
\end{gathered}
$$

then the total mass can be represented by

$$
M_{t}=M_{m}+\gamma M_{t}
$$

where the factor $\gamma$ represents the fraction of $M_{t}$ equal to the equivalent mass of all energies right after the first few seconds of "creation", when the exchange between mass and energy had become negligible [9]. Assuming energy drives the expansion and considering the conservation of the total energy yield

$$
E(t)=M_{r}\left(t_{i n i}\right) c^{2}-c^{2} M_{r}(t)
$$

where $E(t)$ represents the available energy at the time $t$, $M_{r}(t)$ represents the equivalent mass of all the rest of the energies, and $\mathrm{c}$ is the speed of light. The rate of change of this energy is given by

$$
\dot{E}(t)=-c^{2} \dot{M}_{r}(t)
$$

where dot represents differentiation with respect to time $t$. This rate of change of energy can also be represented by the product of the expansion velocity and the rate of change of momentum, i.e,

$$
\dot{E}(t)=\left[\dot{R}(t) \sqrt{1-\left(\frac{\dot{R}(t)}{c}\right)^{2}}\right]\left\{\frac{d}{d t}\left[M_{r}(t) \dot{R}(t) \sqrt{1-(\dot{R}(t) / c)^{2}}\right]\right\}
$$

where $\dot{R}(t)$ represents the expansion velocity and the factor $\sqrt{1-(\dot{R}(t) / c)^{2}}$ represents the effect of time dilation on the rate of expansion. At this stage we are dealing with the total mass and the total equivalent mass of energy. The masses are not affected by the effects of length scale. However, one must consider the effects of length scale on both the rate of expansion and on the definitions of mass and energy densities. To avoid the enigmas associated with the cosmological constant and/or the vacuum energy, we have considered an alternative approach by directly including the correction factor $\sqrt{1-(\dot{R}(t) / c)^{2}}$ for the relativity effect. In equation (7) the effect of the gravitational time dilation is assumed to be negligible compared to the effect of time dilation due to the expansion rate. The effects of length scale on energy densities will be addressed in the next section. Carrying out the differentiation, the above equation can be presented as

$$
\dot{E}(t)=\left[\dot{R}(t) \sqrt{1-\left(\frac{\dot{R}(t)}{c}\right)^{2}}\right]\left\{\begin{array}{c}
\dot{M}_{r}(t)\left[\dot{R}(t) \sqrt{1-\left(\frac{\dot{R}(t)}{c}\right)^{2}}\right]+ \\
M_{r}(t) \frac{d}{d t}\left[\dot{R}(t) \sqrt{1-(\dot{R}(t) / c)^{2}}\right]
\end{array}\right\}
$$

Equating the energy rate from equation (6) to that in equation (8) yields the following differential equation:

$$
\frac{\dot{M}_{r}(t)}{M_{r}(t)}=-\frac{\left[\frac{\dot{R}(t)}{c} \sqrt{1-(\dot{R}(t) / c)^{2}}\right] \frac{d}{d t}\left[\frac{\dot{R}(t)}{c} \sqrt{1-(\dot{R}(t) / c)^{2}}\right]}{1+\left[\frac{\dot{R}(t)}{c} \sqrt{1-(\dot{R}(t) / c)^{2}}\right]^{2}}
$$

Integrating both sides of the above equation from the initial time to the present time, $T_{0}$, yields

$$
M_{r}\left(T_{0}\right)=M_{r}\left(t_{i n i}\right) e^{\chi\left(\frac{\dot{R}\left(T_{0}\right)}{c}\right)}=\gamma M_{t} e^{\chi\left(\frac{\dot{R}\left(T_{0}\right)}{c}\right)}
$$

where $\dot{R}(0)$ is assumed to be zero and

$$
\chi\left(\frac{\dot{R}\left(T_{0}\right)}{c}\right)=-\frac{1}{2} \operatorname{Ln}\left\{1+\left[\frac{\dot{R}\left(T_{0}\right)}{c} \sqrt{1-\left(\dot{R}\left(T_{0}\right) / c\right)^{2}}\right]^{2}\right\}
$$

The equivalent mass $M_{r}\left(T_{0}\right)$ is moving with the speed of light; it represents a total kinetic energy given by

$$
E_{k}\left(T_{0}\right)=\frac{c^{2}}{2} M_{r}\left(T_{0}\right)=\frac{c^{2}}{2} \gamma M_{t} e^{\chi\left(\frac{\dot{R}\left(T_{0}\right)}{c}\right)}
$$

Now considering equations (2) and (10), the change in the kinetic energy up to the present time $T_{0}$ is given by

$$
\Delta E_{k}\left(T_{0}\right)=\frac{c^{2}}{2} \gamma M_{t}-\frac{c^{2}}{2} M_{r}\left(T_{0}\right)=\frac{c^{2}}{2} \gamma M_{t}\left[1-e^{\chi\left(\frac{\dot{R}\left(T_{0}\right)}{c}\right)}\right]
$$

Assuming $\Delta E_{k}\left(T_{0}\right)$ is the total kinetic energy which is imparted to the mass, $M_{m}$, then, including the effect of time dilation on the rate of expansion, the total imparted kinetic energy to the mass $M_{m}$ becomes

$$
\Delta E_{k}\left(T_{0}\right)=M_{m} \frac{\left(\dot{R}\left(T_{0}\right)\right)^{2}\left(1-\left(\frac{\dot{R}\left(T_{0}\right)}{c}\right)^{2}\right)}{2}=\gamma M_{t}\left[1-e^{\chi\left(\frac{\dot{R}\left(T_{0}\right)}{c}\right)}\right] \frac{c^{2}}{2}
$$

Substitution for $M_{m}$ from relation (3) back into the above equation yields

$$
\Delta E_{k}\left(T_{0}\right)=(1-\gamma) M_{t} \frac{\left(\dot{R}\left(T_{0}\right)\right)^{2}\left(1-\left(\frac{\dot{R}\left(T_{0}\right)}{c}\right)^{2}\right)}{2}=\gamma M_{t}\left[1-e^{\chi\left(\frac{\dot{R}\left(T_{0}\right)}{c}\right)}\right] \frac{c^{2}}{2}(15)
$$

Solving the above equation for $\gamma$ yields 


$$
\gamma=\left[1+\frac{1-e^{\left.\chi\left(\frac{\dot{R}\left(T_{0}\right)}{c}\right)\right]}}{\left(\dot{R}\left(T_{0}\right) / c\right)^{2}\left[1-\left(\dot{R}\left(T_{0}\right) / c\right)^{2}\right]}\right]^{-1}
$$

$E_{k}\left(T_{0}\right)$ in equation (12) only represents the total kinetic energy. Substitution for $\gamma$ from the above equation back into equation (10) yields the expression for the total nondimensioned energy as

$e_{t}\left(T_{0}\right)=\frac{E_{t}\left(T_{0}\right)}{c^{2} M_{t}}=\left[1+\frac{1-e^{\chi\left(\frac{\dot{R}\left(T_{0}\right)}{c}\right)}}{\left(\dot{R}\left(T_{0}\right) / c\right)^{2}\left[1-\left(\dot{R}\left(T_{0}\right) / c\right)^{2}\right]}\right]^{-1} e^{\chi\left(\frac{\dot{R}\left(T_{0}\right)}{c}\right)}$

If the universe is in the state of dynamic equilibrium then $e_{t}\left(T_{0}\right)$ must be an extremum at the present time, $T_{0}$. From the above equation, as can also be seen from its plot in Figure (1), we find that the extremum of $e_{t}\left(T_{0}\right)$ is a minimum, and it occurs at the value of $T_{0}$ at which

$$
\frac{\dot{R}\left(T_{0}\right)}{c}=\sqrt{1 / 2}
$$

The fact that the extremum of the total energy, $e_{t}\left(T_{0}\right)$, is a minimum implies that the universe is in a state of stable dynamic equilibrium at the present time, $T_{0}$. Substitution of the above value of $\frac{\dot{R}\left(T_{0}\right)}{c}$ back into equation (11) yields the value of $\chi$ as

$$
\chi=\chi\left(\frac{\dot{R}\left(T_{0}\right)}{c}\right)=\operatorname{Ln} \frac{2}{\sqrt{5}}=-0.111572
$$

Substitution for $\dot{R}\left(T_{0}\right) / c$ from relation (18) back into equation (16) yields the value of $\gamma$ as

$$
\gamma=\frac{1}{61}(25+8 \sqrt{5})=0.703091
$$

In the next section it will be shown that the factor $\gamma$ represents the total energy density parameter.

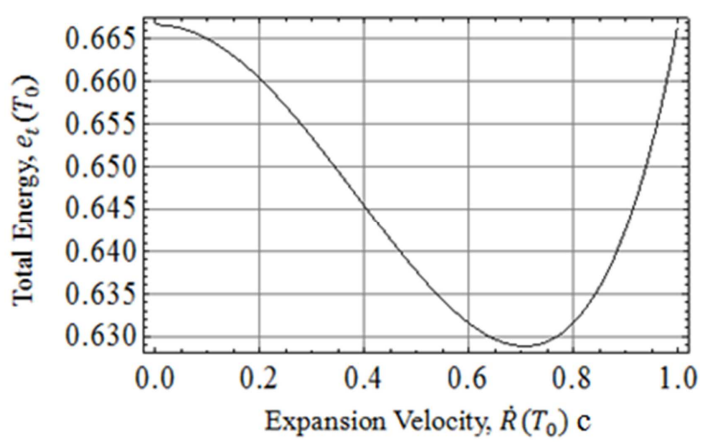

Figure 1. Variation of Total energy versus expansion velocity.

\section{Components of Density Parameter}

As was mentioned before, the length scale affects the definitions of mass and energy densities. To define the mass and energy densities, let $V_{m}$ represent the volume of the mass, $M_{m}$. According to the conservation of mass, $M_{m}=\rho_{m} V_{m}=$ constant, and according to the constancy of the number of photons $M_{r}=\rho_{r} V_{r}=\rho_{r} V_{m}^{4 / 3}=$ constant [10]. Because of the assumption of homogeneous distribution of mass and energy, the mass $M_{m}$ and the equivalent masses $M_{r}$ and $\Delta M_{r}$ would have to be distributed uniformly throughout a unique volume, $V_{\text {univ }}$. Therefore the mass density can be represented by

$$
\rho_{m}(t)=\frac{M_{m}}{V_{\text {univ }}}=\frac{(1-\gamma) M_{t}}{V_{\text {univ }}}=(1-\gamma) \rho_{t}
$$

where $\rho_{t}=\frac{M_{t}}{V_{\text {univ }}}$ represents the universe's total density. Then based on equation (10) the equivalent mass density of the energy density is given by

$$
\rho_{r}(t)=\frac{M_{r}(t)}{V_{\text {univ }}}=\frac{\gamma M_{t}}{V_{\text {univ }}} e^{\chi\left(\frac{\dot{R}(t)}{c}\right)}=\gamma \rho_{t}(t) e^{\chi\left(\frac{\dot{R}(t)}{c}\right)}
$$

Based on equation (13), by the time $t$, the change in the equivalent mass density of energy density is given by

$$
\Delta \rho_{r}(t)=\frac{\gamma M_{t}}{V_{\text {univ }}}\left(1-e^{\chi\left(\frac{\dot{R}(t)}{c}\right)}\right)=\gamma \rho_{t}(t)\left(1-e^{\chi\left(\frac{\dot{R}(t)}{c}\right)}\right)
$$
by

Therefore the total density at the present time, $T_{0}$, is given

$$
\rho_{t_{0}}=\rho_{m 0}+\rho_{r 0}+\Delta \rho_{r 0}
$$

where the subscript 0 denotes present-time value. Then the present-time values of density parameters are defined by:

$$
\begin{gathered}
\Omega_{m 0}=\frac{\rho_{m 0}}{\rho_{c r_{0}}} \\
\Omega_{r 0}=\frac{\rho_{r 0}}{\rho_{c r_{0}}} \\
\Delta \Omega_{r 0}=\frac{\Delta \rho_{r 0}}{\rho_{c r_{0}}}
\end{gathered}
$$

where $\rho_{c r_{0}}=\frac{3 H_{0}^{2}}{8 \pi G}$ represents the critical density of the universe at the present time. Assuming the universe to be at its critical density at the present time, $t_{0}=T_{0}$, that is $\rho_{t_{0}}=\rho_{c r_{0}}$, then the total density parameter, $\Omega_{0}$, at the present time is given by

$$
\Omega_{0}=\Omega_{m 0}+\Omega_{r 0}+\Delta \Omega_{r 0}=\frac{\rho_{t_{0}}}{\rho_{c r_{0}}}=1
$$

Considering relations (22) and (23) together with relations (26) and (27), it can be shown that $\gamma$ represents the total energy density parameter. Thus

$$
\gamma=\Omega_{r 0}+\Delta \Omega_{r 0}
$$

In the next section, it will be shown that the total energy, as represented by the total energy density parameter, $\gamma$, creates the pressure which is responsible for the expansion of space.

\section{Analytical Estimate of the Hubble Constant}

According to the equation of state, the magnitude of the 
radiation pressure is given by $P_{r}=\frac{1}{3} c^{2} \rho_{r}$, Misner, et al. [10]. Thus substitution for $\rho_{r}(t)$ from relation (22) yields

$$
P_{r}=\frac{1}{3} c^{2} \rho_{r}(t)=\frac{1}{3} c^{2} \gamma \rho_{t}(t) e^{\chi\left(\frac{\dot{R}(t)}{c}\right)}
$$

Assuming the universe to be at its critical density at the present time, the above pressure can be represented by

$$
\begin{gathered}
P_{r 0}=\frac{1}{3} c^{2} \gamma \rho_{t_{0}} e^{\chi\left(\frac{\dot{R}\left(T_{0}\right)}{c}\right)}=\frac{1}{3} c^{2} \gamma\left(\frac{\rho_{t_{0}}}{\rho_{c r_{0}}}\right) \frac{3 H_{0}^{2}}{8 \pi G} e^{\chi\left(\frac{\dot{R}\left(T_{0}\right)}{c}\right)}= \\
c^{2} \gamma \frac{H_{0}^{2}}{8 \pi G} e^{\chi\left(\frac{\dot{R}\left(T_{0}\right)}{c}\right)}
\end{gathered}
$$

In a uniform acceleration field, at any point $R$, the radiation pressure can be equivalently represented by

$$
P_{r}=-\ddot{R}(t)\left(\frac{\text { unit mass }}{\text { unit area }}\right)
$$

where $\ddot{R}$ represents the acceleration that yields the above equality. According to Einstein's equivalence principle, the acceleration at any point, in a uniform acceleration field, is equivalent to a gravitational acceleration at that point. Therefore $\ddot{R}$ also represents the gravitational acceleration in the assumed flat universe. To find the acceleration at a point $R$, for the flat universe with zero cosmological constant, using conservation of energy or the FRW equation one obtains

$$
\left(\frac{\dot{R}(t)}{R(t)}\right)^{2}=\frac{2 M_{t} G}{[R(t)]^{3}}
$$

But $\frac{M_{t} G}{[R(t)]^{2}}=-\ddot{R}(t)$. Therefore

$$
\ddot{R}(t)=-\frac{M_{t} G}{[R(t)]^{2}}=-\frac{1}{2} \frac{\dot{R}(t)}{R(t)} \dot{R}(t)
$$

Correcting the velocity in the above relation for the effect of time dilation yields

$$
\ddot{R}(t)=-\frac{1}{2}\left\{\frac{\left[\dot{R}(t) \sqrt{1-\left(\frac{\dot{R}(t)}{c}\right)^{2}}\right]}{R(t)}\right\}\left[\dot{R}(t) \sqrt{1-\left(\frac{\dot{R}(t)}{c}\right)^{2}}\right]
$$

where in the above relation the velocity, $\left(\dot{R}(t) \sqrt{1-\left(\frac{\dot{R}(t)}{c}\right)^{2}}\right)=V_{\text {cor }}(t)$, represents the expansion velocity corrected for the effect of time dilation. Thus the present-time value of the gravitational acceleration at the point $R$ is given by

$$
\ddot{R}\left(T_{0}\right)=-\frac{1}{2} H_{0} V_{\text {cor }}\left(T_{0}\right)=-\frac{1}{2} H_{0} \dot{R}\left(T_{0}\right) \sqrt{1-\left(\frac{\dot{R}\left(T_{0}\right)}{c}\right)^{2}}
$$

where

$$
H_{0}=\frac{V_{c o r}\left(T_{0}\right)}{R\left(T_{0}\right)}=\frac{V_{c o r}\left(T_{0}\right)}{T_{0} V_{c o r}\left(T_{0}\right)}=\frac{1}{T_{0}}=\left(\dot{R}\left(T_{0}\right) \sqrt{1-\left(\frac{\dot{R}\left(T_{0}\right)}{c}\right)^{2}}\right) / R\left(T_{0}\right)
$$

is the Hubble constant corrected for the effect of time dilation. The negative sign in equation (32) means that the direction of radiation pressure is opposite to the direction of the gravitational acceleration. This implies that the expansion is taking place at an accelerating rate. This is consistent with the finding that the rate of expansion is accelerating [11, 12 , 13, 14]. Substitution for $\ddot{R}(T)$ from the above relation back into equation (32) yields

$$
P_{r 0}=\frac{1}{2} H_{0} \dot{R}\left(T_{0}\right) \sqrt{1-\left(\frac{\dot{R}\left(T_{0}\right)}{c}\right)^{2}}\left(\frac{\text { unit mass }}{\text { unit area }}\right)
$$

The right side of the above equation involves the kinematic terms, while the left side represents the kinetics of the system. Thus this expression represents the relation between the geometry and the kinetics of the system. Equating the pressure $P_{r 0}$ from the above relation to $P_{r 0}$ in equation (31) yields

$$
c^{2} \gamma \frac{H_{0}^{2}}{8 \pi G} e^{\chi\left(\frac{\dot{R}\left(T_{0}\right)}{c}\right)}=\frac{1}{2} H_{0} \dot{R}\left(T_{0}\right) \sqrt{1-\left(\frac{\dot{R}\left(T_{0}\right)}{c}\right)^{2}}\left(\frac{\text { unit mass }}{\text { unit area }}\right)
$$

Solving the above relation yields the present-time value for the Hubble constant as

$$
H_{0}=4 \pi \frac{G}{c^{2} \gamma} e^{-\chi\left(\frac{\dot{R}\left(T_{0}\right)}{c}\right)} \dot{R}\left(T_{0}\right) \sqrt{1-\left(\frac{\dot{R}\left(T_{0}\right)}{c}\right)^{2}}\left(\frac{\text { unit mass }}{\text { unit area }}\right)
$$

Recalling from relation (18) that $\dot{R}\left(T_{0}\right)=\sqrt{1 / 2} c$ and substituting it into the above equation yields

$$
H_{0}=\beta \frac{G}{c}
$$

where the parameter $\beta$ is given by

$$
\beta=\frac{2 \pi e^{-\chi\left(\frac{\dot{R}\left(T_{0}\right)}{c}\right)}}{\gamma}\left(\frac{\text { unit mass }}{\text { unit area }}\right)
$$

Thus substitutions of the values for $\chi\left(\frac{\dot{R}\left(T_{0}\right)}{c}\right)$ and $\gamma$, as given by relations (19) and (20), back into relations (41a) and (41b) yield the present-time values for the parameter $\beta$ and the Hubble constant as

$$
\begin{gathered}
\beta=(5 \sqrt{5}-8) \pi \mathrm{kg} / \mathrm{m}^{2}=9.99133 \mathrm{~kg} / \mathrm{m}^{2} \\
H_{0}=2.23489 \times 10^{-18} \mathrm{~s}^{-1}=69.05398 \mathrm{~km} \mathrm{~s}^{-1} \mathrm{Mpc}^{-1}
\end{gathered}
$$

Therefore, the value of the present time, $T_{0}$ (commonly called age of the universe) is given by $T_{0}=H_{0}^{-1}=$ 14.1628 GY. Also, equation (35) corrected for sign yields the present-time value of the expansion acceleration as

$$
\ddot{R}\left(T_{0}\right)=\frac{1}{2} H_{0} \dot{R}\left(T_{0}\right) \sqrt{1-\left(\frac{\dot{R}\left(T_{0}\right)}{c}\right)^{2}}=\frac{1}{4} H_{0} c
$$

Because $\mathrm{c}$ and $\mathrm{G}$ are universal constants, at any time, whether past, present, or future, equation (41a) sets the same definite limit on how far one can look back in time. It also extends the meaning of the cosmological principle from "the universe looks the same wherever it is observed" to "the universe looks the same wherever and whenever it is observed". 


\section{Checks and Comparisons}

In this section we check the quality of the proposed analytical approach. Using Mattig's formula, Peebles [15], it can be shown that the luminosity distance at the present time is given by

$$
D_{L}=\frac{c}{H_{0}} \frac{2\left[\Omega_{0} \mathrm{z}+\left(\Omega_{0}-2\right)\left(\sqrt{1+\Omega_{0} \mathrm{z}}-1\right)\right]}{\Omega_{0}^{2}}
$$

Assuming a flat universe at its critical density at the present time, then according to relation (28) the total density parameter $\Omega_{0}=1$, and the above equation simplifies to

$$
D_{L}=\frac{2 c}{H_{0}}(1+z-\sqrt{1+z})
$$

The distance modulus and its K-correction [9] are

$$
\begin{gathered}
\mu=5 \log _{10} D_{L}+K(z) \\
K(z)=-2.5(\alpha-1) \log _{10}(1+z)
\end{gathered}
$$

where $D_{L}$ is in $10 p c$ units. The $K$-correction serves to compensate for the difference in the band in which the flux is emitted and the band in which it is observed. As the analytical approach involves no aperture correction, the value of $\alpha$ in the above equation is zero. Thus

$$
K(z)=2.5 \log _{10}(1+z)=5 \log _{10} \sqrt{1+z}
$$

If one applies this correction to the luminosity distance, then the corrected luminosity distance can be represented by

$$
\left(D_{L}\right)_{\text {cor }}=\sqrt{1+z} D_{L}
$$

Thus the distance modulus can be represented by

$$
\mu=5 \log _{10} D_{L}+K(z)=5 \log _{10}\left(D_{L}\right)_{c o r}=5 \log _{10}\left(\sqrt{1+z} D_{L}\right)
$$

To check the efficacy of the proposed analytical estimate of the Hubble constant, we use a set of $557 \mathrm{SNe}$ data, as reported in 2010, in the Union2 Compilation [16], and a set of 394 extragalactic distances to 349 galaxies at cosmological redshifts significantly higher than the Union 2 Compilation, as reported in 2008 by Mador and Steer [17].

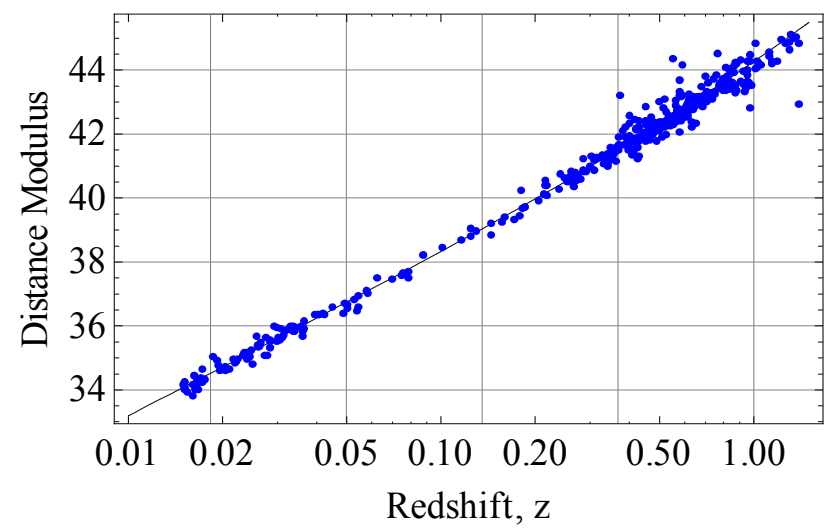

Figure 2. Hubble Diagram, Conformity of the Analytical Curve with the Measured Data. Blue dots represent 557 data points from the Union2 Compilation.

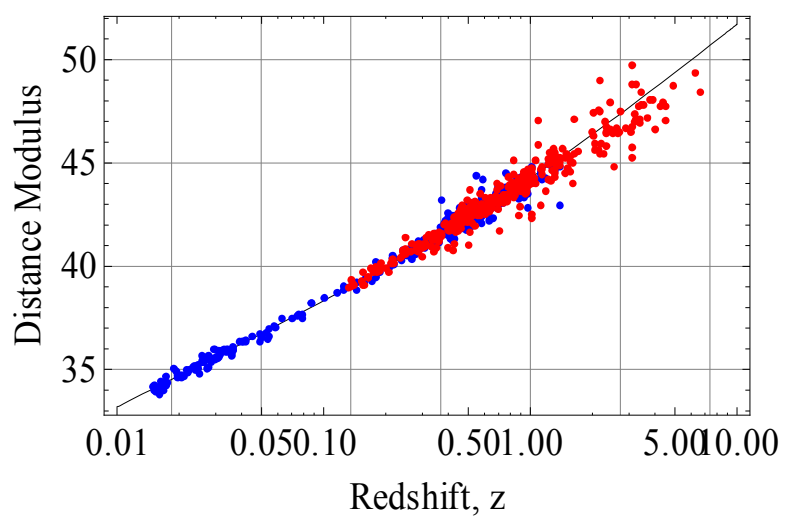

Figure 3. Hubble Diagram, Conformity of the Analytical Curve with the Measured Data. Blue dots represent the 557 data points from the Union2 Compilation. Red dots represent 394 data points from NASA/IPAC Extragalactic Database, NED-4D

Using the value of $\Omega_{0}=1$ as given by equation (28), the calculated value of the Hubble constant of $H_{0}=$ $69.05398 \mathrm{~km} \mathrm{~s}^{-1} \mathrm{Mpc}^{-1}$, and equations (45) and (50), plots of the distance modulus versus redshift are presented in Figures 2 and 3 . The redshift in the union 2 compilation data varies from a low of $z=0.0152$ to a maximum of $z=1.4$. These data are plotted in Figure 2. The redshift in the extragalactic database varies from a low of $z=0.133$ to a maximum of $z=6.6$. These data combined with the union2 data are plotted in Figure 3. These figures confirm the consistency of the analytically derived value of the Hubble constant with the observational data.

\section{Remarks and Conclusions}

Assuming a flat universe with a zero cosmological constant, the value of the Hubble constant is analytically derived to be equal to $69.05398 \mathrm{~km} \mathrm{~s}^{-1} \mathrm{Mpc}^{-1}$. This value is in between the ones estimated by the Nine-Year Wilkinson Microwave Anisotropy Probe (WMAP) and the Megamaser Cosmology Project. IV team. The remarkable consistency of the analytical curve with the observational data, displayed in Figures 2 and 3, justifies the assumptions of the universe being flat and being at its critical density. Also, because the total energy is at its minimum at the present time, the universe as a whole is in a state of stable dynamic equilibrium.

The total energy is constant and its two parts participate in setting up the mechanism for the expansion of space. The part represented by $\Delta \Omega_{r}$ imparts energy to the mass, $\mathrm{M}_{m}$, while the total energy, as represented by the total energy density parameter, $\gamma$, creates the pressure which drives the expansion.

\section{References}

[1] E. Hubble, "A Relation between Distance and Radial Velocity among Extra-Galactic Nebulae," Proceedings of the National Academy of Sciences of the United States of America, 15:3, 168-173 (1929). http://www.pnas.org/content/15/3/168 
[2] L. Wendy, W. L. Freedman, F. B. Madore, " The Hubble Constant," Annual Review of Astronomy and Astrophysics, 48:673-710, (2010).

[3] Tammann, G. A. (2005) "The Ups and Downs of the Hubble Constant," arXiv:astro-ph/0512584v1, Reviews in Modern Astronomy, Volume 19, pp. 1-29, (2008).

[4] N. Falcon, A. Aguirre, "Theoretical Deduction of the Hubble Law Beginning with a MoND Theory in Context of the $\Lambda$ FRW-Cosmology," International Journal of Astronomy and Astrophysics, 4, pp. 551-559, (2014). http://dx.doi.org/10.4236/ijaa.2014.44051

[5] D. Larson, J. Dunkley, G. Hinshaw, E. Komatsu, M. R. Nolta, C. L. Bennett, et al., "Seven-Year Wilkinson Microwave Anisotropy Probe (WMAP) Observations: Sky Maps, Systematic Errors, and Basic Results," Astrophysical Journal Supplement Series, 192:16, pp. 1-19 (2011).

[6] P. A. R. Ade, N. Aghanim, M. I. R. Alves, C. , ArmitageCaplan, M. Amaud, M. Ashdown, et al., "Planck 2013 Results, I. Overview of Products and Scientific Results," http://arxiv.org/abs/1303.5062 v2, pp. 1-49 (2013).

[7] C. L. Bennett, D. Larson, J. L. Weiland, N. Jarosik, G. Hinshaw, N. Odegard, et al., "Nine-Year Wilkinson Microwave Anisotropy Probe (WMAP) Observations: Maps and Results," Astrophysical Journal Supplement Series, 208:20, pp. 1-54 (2013).

[8] M. J. Reid, J. A. Braatz, J. J. Condon, K. Y. Lo, C. Y. Kuo, C. M. V. Impellizzeri, et al. "The Megamaser Cosmology Project IV. A Direct Measurement of the Hubble Constant From UGC 3789," Astrophysical Journal, 767:154, pp. 1-11, (2013).

[9] J. A. Peacock, Cosmological Physics, Cambridge University Press, 1999, pp. 75-77.
[10] C. W. Misner, K. S. Thorne, J. A. Wheeler, Gravitation, W. H. Freeman and Company, San Francisco, 1973, pp. 726-728.

[11] A. G. Riess, "Nobel Lecture: My Path to the Accelerating Universe," Reviews of Modern Physics, Vol. 84, pp. 11651175, (2012).

[12] B. P. Schmidt, "Nobel Lecture: Accelerating Expansion of the Universe through Observation of Distant Supernovae," Reviews of Modern Physics, Vol. 84, pp. 1151-1163, (2012).

[13] S. Perlmutter, "Nobel Lecture: Measuring the Acceleration of the Cosmic Expansion Using Supernovae," Reviews of Modern Physics, Vol. 84, pp. 1127-1149, (2012).

[14] S. Perlmutter, G. Aldering, G. Goldhaber, R. A. Knop, P. Nugent, P. G. Castro, et al. "Measurements of $\Omega$ and $\Lambda$ From 42 High-Redshift Supernovae," The Astrophysical Journal, 517:565-586, (1999).

[15] P. J. E. Peebles, Principles of Physical Cosmology, Princeton University Press 1993, pp. 321.

[16] R. Amanullah, C. Lidman, D. Rubin, G. Aldering, P. Astier, K. Barbary, et al., "Spectra and Hubble Space Telescope Light Curves of Six Types Ia Supernovae at $0.511<\mathrm{z}<1.12$ and the Union2 Compilation," The Astrophysical Journal, 716:712738, (2010), data available at http://supernova.lbl.gov/Union/figur...n2_mu_vs_z.txt

[17] B. F. Mador, I. P. Steer, "NASA/IPAC Extragalactic Database Master List of Galaxy Distances," NED-4D, (2008). http://ned.ipac.caltech.edu/level5/NED4D/ 